\title{
Otomastoiditis tuberculosa: A propósito de un caso
}

JUAN I. JUANET G.*, MIRTA ACUÑA A.**, CARLOS CASAR C.**

\section{Tuberculous Otomastoiditis: A Clinical Case}

Tuberculosis (TB) has been one of the infectious diseases most studied in the history of medicine. In Chile, TB incidence was 13.7 per 100,000 inhabitants in 2008, being the country with the lowest incidence in Latin America. World-wide publications consider tuberculous otitis media (TOM) as a very rare form of TB presentation, constituting 0.05 to $0.9 \%$ of chronic otitis media. Population younger than 15 years old is the most vulnerable, $85 \%$ of the cases belongs to this range of age. Chile has not epidemiological data available on this pathology. The aims of this article are to show a clinical case of TOM and posterior otomastoiditis, in an infant hospitalized in Roberto del Rio Children's Hospital in Santiago de Chile and review the literature about this pathology, in order to raise awareness for doing an opportune diagnosis of this condition.

Key words: Tuberculous otitis media, tuberculosis and otomastoiditis

\section{Resumen}

La tuberculosis (TBC), es una de las patologías infecciosas más estudiadas en medicina. En Chile, la incidencia de TBC en 2008 fue de 13,7 por 100,000 habitantes, situando al país en fase de eliminación y con la menor incidencia en América Latina. Las publicaciones internacionales consideran que la otitis media por TBC (OMT) es una forma de presentación clínica muy infrecuente de TBC con una incidencia que oscila entre un 0,05 y un 0,9\% de las otitis media crónicas. La población más afectada es la menor de quince años, lo que constituye el $85 \%$ de los casos. En Chile no se cuenta con datos epidemiológicos de dicha patología. El objetivo de esta publicación es dar a conocer un caso clínico de OMT complicada con otomastoidistis, en un paciente del hospital de niños Dr. Roberto de Río de Santiago, Chile y revisar la información disponible en la literatura para concientizar a la población médica.

Palabras clave: Otitis media crónica, tuberculosis y otomastoiditis.

\section{Introducción}

La tuberculosis (TBC), es una de las patologías infecciosas más estudiadas en la historia de la medicina, tanto en el pasado como en la era actual, por ser esta un problema de salud pública. Durante gran parte del siglo XX inquietaba su elevada incidencia y mortalidad. Actualmente, el surgimiento de cepas resistentes a las distintas drogas anti-tuberculosas, y el aumento de la frecuencia de dicha enfermedad en los últimos años, es lo que mantiene en alerta a los equipos de salud ${ }^{1}$.

En Chile, la incidencia de TBC en 2008 fue de 13,7 por 100,000 habitantes, situando al país en fase de eliminación y con la menor incidencia en América Latina. Cabe destacar, que es en los menores de quince años donde ha ocurrido el descenso más importante de casos: desde 23,4/100.000 habitantes hace unas décadas hasta

Sin conflicto de intereses. Sin fuente de financimiento. Establecimiento donde se realizó el trabajo: Hospital de Niños Dr. Roberto del Río.

* Hospital Clínico Universidad de Chile.

** Hospital de Niños Dr. Roberto del Río. Santiago, Chile. 
alcanzar actualmente a 2,3/100.0002. La forma de presentación clínica más frecuente en la población pediátrica es la del complejo primario.

La otitis media crónica por tuberculosis (OMT) fue descrita por primera vez en 1853, y fue Eschle quien en 1883 aisló el bacilo en secreción ótica ${ }^{3,4}$. A principios del siglo XX, 3 a $5 \%$ de las otitis medias crónicas eran producidas por TBC con un 9,5\% de ellas presentándose en los menores de 5 años ${ }^{5}$. Uno de los principales mecanismos de transmisión del bacilo al oído medio, se producía por el paso de la leche no pasteurizada a través de la trompa de Eustaquio ${ }^{5}$.

La OMT es una forma de presentación clínica muy infrecuente de TBC, cuya incidencia actualmente oscila entre 0,05 a $0,9 \%$ de las otitis media crónica $^{3-5}$. En Chile, no se cuenta con suficientes datos epidemiológicos de dicha patología por no existir casos descritos en la literatura médica nacional. Como se mencionó anteriormente, la población más afectada es la menor de quince años, lo que constituye el $85 \%$ de los casos ${ }^{5}$.

La disminución de esta forma clínica de TBC, se debe a varios factores, tales como: los progresos en el área de la salud pública, la vacuna del bacilo Calmette Guerin, la disponibilidad de medicamentos anti-TBC y el mejoramiento de la calidad de vida ${ }^{4}$. Por otra parte, ya que los signos y síntomas de la OMT son difíciles de diferenciar de la otitis media crónica causada por otros agentes, y que la frecuencia de esta patología ha decaído, se ha producido una disminución importante de la sospecha clínica, con el riesgo de un diagnóstico tardío con las implicancias y complicaciones que este conlleva. A modo de ejemplo podemos citar, la hipoacusia, la cual puede llegar a ser permanente. ${ }^{7}$

\section{Caso clínico}

Lactante mayor de nacionalidad chilena, tercer hijo de una familia disfuncional, con un padre en prisión por robo y una madre de carácter agresivo, severo, que no cumple adecuadamente con las indicaciones médicas. El paciente presenta antecedentes mórbidos de bronconeumonías repetidas, con hospitalizaciones reiteradas en unidades de cuidados intensivos desde los 11 meses de edad hasta el año cuatro meses.

$\mathrm{Al}$ año cinco meses se hospitaliza nuevamente por un síndrome febril prolongado, con signología pulmonar húmeda y requerimiento adicional de oxígeno, que persiste por 30 días, (basándose en los resultados alterados de repetidas saturometrías).
Se realizan radiografías de tórax las cuales no muestran cambios significativos con respecto a sus placas previas, donde sí se observan lesiones residuales, como infiltrado inflamatorio intersticial bilateral y una atelectasia del lóbulo inferior izquierdo. Los exámenes de laboratorio como el hemograma, los hemocultivos, los urocultivos y el cultivo de secreción traqueal no arrojan resultados compatibles con una infección bacteriana. Las inmunofluorescencias de secreción nasofaríngea para virus respiratorios dan resultados negativos, y un lavado broncoalveolar descarta una infección pulmonar por microorganismos atípicos como citomegalovirus, hongos y mycobacterias. Sin embargo, llama la atención una velocidad de eritrosedimentación de $100 \mathrm{~mm} /$ hora, (tomada en dos ocasiones), y una proteína $\mathrm{C}$ reactiva mayor a $100 \mathrm{mg} / \mathrm{L}$.

$\mathrm{Al}$ reexaminar al paciente, se pesquisa una otorrea seropurulenta proveniente del oído izquierdo. Por sospecha de otitis media aguda supurada se inician antibióticos sistémicos de primera línea, amoxicilina por diez días y luego de segunda, amoxicilina-ácido clavulánico, con respuesta parcial al tratamiento.

A los veinte días de estar hospitalizado y persistir febril, se procede a estudios de imágenes. Se realizan un ecocardiograma, sin signos de una endocarditis, una ecografía abdominal normal, un cintigrama óseo sin foco inflamatorio osteoarticular y una tomografía computada de cavidades paranasales que no arroja resultados alterados.

Por requerir de oxígeno complementario, presentar signología pulmonar húmeda, marcadores inflamatorios elevados y una radiografía de tórax alterada, se sospecha posible TBC pulmonar. Se toma un PPD con resultado positivo de $15 \mathrm{~mm}$. Sin embargo, el estudio con baciloscopías y cultivos de Köch de secreción traqueal y aspiración gástrica resulta negativo. Se completa el estudio con Quantiferón Gold TB (interferón gama para TBC) informándose como positivo. Así, se confirma el diagnóstico de TBC por lo que se inicia tratamiento triasociado con isoniazida, rifampicina y pirazinamida. Con esto cede la fiebre y se suprimen los requerimientos adicionales de oxígeno en forma concomitante.

Por antecedentes de bronconeumonias graves a repetición, incluida la TBC pulmonar actual, se efectuó un estudio de VIH, previo al alta, con dos resultados negativos, y se realizan pruebas de inmunidad humoral y celular, cuyos resultados están dentro de los rangos normales. También, el paciente es evaluado por otorrinolaringólogo, quien confirma una otitis media crónica supurada. En el contexto de paciente con TBC pulmonar, 
se sospecha etiología tuberculosa, por lo que se sugiere una tomografía computada del peñasco izquierdo para descartar otomastoiditis por TBC. Sin embargo, la madre pierde la cita del examen y éste no se realiza.

Dos meses más tarde, al año ocho meses de edad, el lactante se hospitaliza nuevamente por un cuadro convulsivo febril complejo con compromiso de conciencia. Se realiza punción lumbar con salida de líquido cefalorraquídeo alterado por pleocitosis de 584 células $/ \mathrm{mm}^{3}$ con predominio mononuclear de $88,6 \%$, una glucosa normal de $48 \mathrm{mg} / \mathrm{dL}$, proteínas con leve elevación de $51 \mathrm{mg} /$ $\mathrm{dL}$, adenosindeaminasa normal y baciloscopía negativa.

Por sospecha de infección del sistema nervioso central se traslada a unidad de cuidados intensivos. Se aprecia aumento de volumen blando retroauricular izquierdo. Se realiza una tomografía computada cerebral que muestra signos inespecíficos de inflamación focal en lóbulo temporal izquierdo, y una ocupación de todas las celdillas mastoideas izquierdas con esclerosis y destrucción ósea. (Figura 1). Esto sugiere una ostomastoiditis crónica por lo que se inicia tratamiento empírico de amplio espectro con ceftazidima, amikacina, vancomicina, metronidazol y aciclovir para así cubrir Pseudomonas aeruginosa, Staphylococcus aureus, anaerobios y etiología herpética. El electroencefalograma no muestra actividad epileptiforme ni signos que indiquen infección herpética. Se toma cultivo de secreción ótica izquierda con resultado positivo

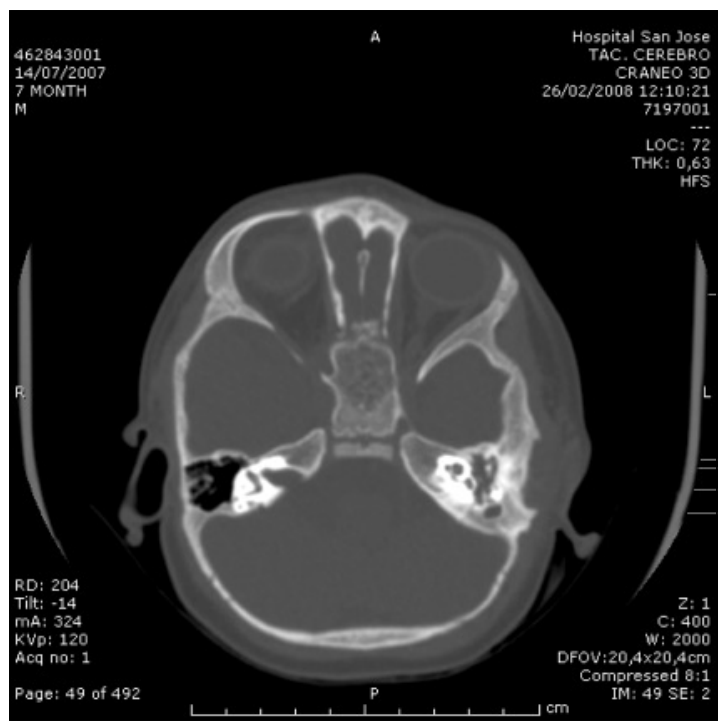

Figura 1. Ocupación de las celdillas mastoideas izquierdas con destrucción ósea. Signos sugerentes de otomastoiditis crónica izquierda. para Pseudomonas aeruginosa. El paciente evoluciona en buenas condiciones generales y sin complicaciones. Se rescatan cultivo corriente, de Koch y reacción de polimersasa en cadena (PCR) para herpes virus simplex 1 y 2 y herpes 6 de líquido cefalorraquídeo negativos, por lo que se mantiene solamente la antibioterapia para cubrir Pseudomonas aeruginosa por 21 días, suspendiéndose el resto. Posteriormente, se completa el estudio con una resonancia nuclear magnética cerebral con resultado normal. Como unidad diagnóstica, se concluye que el paciente presenta una otomastoiditis crónica, posiblemente de etiología tuberculosa, y una meningitis a líquido claro, causado probablemente por contigüidad del proceso inflamatorio mastoideo.

Un mes más tarde de su ingreso, en conjunto con equipo otorrinolaringológico, se decide intervenir quirúrgicamente al paciente, para confirmación diagnóstica y tratamiento de su mastoiditis crónica. Se realiza una mastoidectomía izquierda y biopsia del tejido, con una macroscopía cuyo resultado muestra un proceso inflamatorio crónico, granulomatoso, necrotizante y caseificante con áreas de actividad inflamatoria aguda supurada. La microscopía se caracteriza por un infiltrado inflamatorio mixto, linfoplasmocitario de polimorfonucleares neutrófilos, con granulomas y células gigantes multinucleadas de tipo Langhans. El análisis anatomopatológico concluye aspecto morfológico compatible con TBC.

Las baciloscopías, y los cultivos de secreción ótica, y de biopsia arrojan resultados negativos. A pesar de no aislar el bacilo se establece diagnóstico de otomastoiditis crónica por TBC por la compatibilidad anatomopatológica.

En forma retrospectiva, se confirma con la madre, y luego con el centro de atención primaria correspondiente, que no se cumplió con el tratamiento anti-tuberculoso, ya que ella no se presentó en el centro de salud con regularidad para administrarle los medicamentos a su hijo. Por ello, y por presentar como complicación una otomastoiditis, se decide re-iniciar esquema con adición de estreptomicina por 50 dosis en fase diaria, lo que se realiza en forma intrahospitalaria. Luego se cambia a rifampicina e isoniazida de administración bisemanal por 40 dosis en forma ambulatoria, bajo supervisión estricta de la atención primaria de salud.

Posterior al término del tratamiento antituberculoso, se controla al paciente en forma ambulatoria estando asintomático del punto de vista tanto ótico como pulmonar por tener tímpanos y conductos auditivos indemnes y no volver a requerir de oxígeno complementario. 


\section{Discusión}

A pesar de la disminución de la incidencia de TBC, durante los últimos años, se ha observado un aumento de los casos en forma mundial. Esto se debe a distintas causas, siendo algunas de ellas la epidemia del SIDA, el incremento de las migraciones desde zonas endémicas, el hacinamiento en distintas áreas geográficas, y al control parcial de la administración de los medicamentos por parte de las autoridades de salud. ${ }^{3,7}$ En este caso, el paciente, inicialmente no recibió en forma regular su tratamiento anti-tuberculoso. Según las normas de nuestro país, se debió haber notificado a las autoridades sanitarias. Sin embargo, por el hecho de atenderse irregularmente en los diferentes servicios de salud, (atención primaria y terciaria), la notificación no pudo ser hecha en forma correcta.

En un paciente con otitis media crónica se debe mantener siempre una sospecha de OMT, especialmente si fracasa el tratamiento antibiótico, si ha tenido algún contacto de TBC, o si cursa con una TBC pulmonar o extrapulmonar. Esta última asociación se observa entre el $40 \%$ a $65 \%$ de los $\operatorname{casos}^{3,5}$. En el caso del lactante de este artículo, se concluyó que tenía factores de riesgo epidemiológicos para TBC, por visitar con frecuencia a su padre en prisión. En la población penitenciaria, por las condiciones de hacinamiento, aumentan las posibilidades de contagio por TBC.

Las vías de transmisión posibles son diversas, pero la más frecuente es la hematógena secundaria a una TBC miliar. Otras posibles son las que se producen en forma directa, es decir, desde el nasopharynx a través de la trompa de Eustaquio, por extensión linfática, por forma externa debido a perforación timpánica, por extensión directa de estructuras adyacentes, como el sistema nervioso central, y por infección congénita, ya sea por vía placentaria o la que ocurre en el canal del parto ${ }^{1,3,6,8}$.

Con respecto a la clínica, la tríada clásica descrita desde el descubrimiento de la OMT ha sido una otorrea indolora, perforación timpánica y parálisis facial. Es necesario destacar, que actualmente, se presenta en forma más heterogénea, caracterizada por una evolución lenta e insidiosa de otorrea serosa, que puede tornarse purulenta por sobreinfección bacteriana, con o sin otalgia, e hipoacusia al momento del diagnóstico $0^{4,5,7,9}$.

En la otoscopía es posible confirmar la otorrea serosa o purulenta proveniente del oído medio, visualizándose signos que sugieren infección por TBC: una membrana timpánica gruesa e hiperémica, perforación timpánica única o múltiple, granulaciones pálidas, hiperémicas y friables del oído medio, destrucción de los huesesillos, erosión del hueso cortical mastoideo y compromiso de la vaina del nervio facial. En la región periauricular se pueden encontrar adenopatías anteriores y posteriores, así como también en la zona cervical ${ }^{4,5,6}$. En este paciente se manifiesta por su otorrea purulenta persistente.

La confirmación etiológica de TBC para la OMT es difícil. Primero, en niños infectados con el bacilo de Köch la población bacilar es mínima, por lo que suelen tener baciloscopías de secreción traqueal y de aspirado gástrico negativas. Segundo, como en otras formas extrapulmonares, el oído medio tiene baja concentración de bacilos, y además el uso frecuente de gotas óticas con neomicina, u otros antibióticos, dan como resultado análisis bacteriológicos de secreciones y tejido histológico de bajo rendimiento ${ }^{4,9,10}$. En este caso específico, al paciente no se le suministró ningún tipo de gotas. Tercero, las características propias del crecimiento lento de este agente, y la interferencia de su desarrollo por otras variedades de bacterias, hacen que la probabilidad de baciloscopía y cultivo positivo en secreción ótica sea menor al $20 \%$ y entre un 5 a un $35 \%$ respectivamente ${ }^{3,6,7}$. Este paciente presentó una coinfección con Pseudomonas aeruginosa.

La anatomía patológica cumple un rol primordial en relación al diagnóstico etiológico de esta enfermedad. Una histología concordante con una infección por TBC, con un tejido granulomatoso con células epiteloídeas y células gigantes de Langhans con áreas caseosas y necróticas, junto a una sospecha clínica, son suficientes para confirmar el diagnóstico, independientemente a los resultados de los cultivos, teniendo en cuenta el mal rendimiento de éstos. En cuanto al lactante en cuestión, fue el aspecto morfológico y una histología concordante con TBC la que puso el sello al diagnóstico, ya que el bacilo nunca pudo ser aislado.

Otra alternativa disponible para el diagnóstico, es la técnica de reacción de polimerasa en cadena (PCR) para el análisis genético del bacilo en secreción ótica o biopsia de tejido, con una elevada sensibilidad y especificidad. Sin embargo, un resultado negativo no descarta infección por $\mathrm{TBC}^{1}$.

La radiología es fundamental, ya que no sólo permite orientarse hacia un diagnóstico, sino que además puede evidenciar posibles complicaciones. El método de elección es la tomografía computada, donde se puede objetivar opacificación del oído medio y mastoides, esclerosis de la corteza mastoidea, aumento de densidad y radioluscencia de la corteza mastoidea si existe 
resorción ósea, destrucción de huesesillos y destrucción ósea mastoidea o del hueso petroso ${ }^{10,11}$.

En la formulación del diagnóstico de toda otitis media crónica se deben tener presente, y descartar, como posibles causas, la infección por hongos, un colesteatoma, histiocitosis, granulomatosis de Wegener, granulomatosis eosinofílica, sífilis y sarcoidosis ${ }^{5,9,12}$.

Las posibles complicaciones de la OMT son numerosas. La más frecuente es la hipoacusia, presentándose en el $90 \%$ de los casos. Ésta puede ser de tipo conductiva en un $90 \%$, sensorioneural en un $8 \%$, y mixta en un $2 \%{ }^{6,9}$. La reversibilidad de ésta, y la severidad de sus secuelas, están directamente relacionadas con el tiempo de inicio del tratamiento, lo que refuerza una vez más la importancia de una sospecha y de un diagnóstico precoz. La parálisis periférica del nervio facial, otra complicación frecuente, se produce por compresión o por infiltración de su vaina. Se observa en un $16 \%$ hasta un $35 \%$ de los casos y es reversible. Otras complicaciones son la necrosis de la cadena osicular, la otomastoiditis, el secuestro y destrucción ósea de la mastoides, y del hueso petroso, la fístula retroauricular y el compromiso de la articulación témporomandibular. Entre las complicaciones intracraneanas es importante mencionar: meningitis, tuberculoma, abscesos cerebrales, parálisis múltiple de pares craneales, laberintitis, y la trombosis lateral del seno sigmoideo ${ }^{4-7,13}$. Este paciente no presentó parálisis facial, pero sí otomastoiditis, por lo que debió ser intervenido quirúrgicamente.

El tratamiento de la OMT, como ha sido mencionado anteriormente, puede ser conservador como también quirúrgico. El esquema antibiótico indicado es el primario simplificado de TBC para formas pulmonares o extrapulmonares, el cual consiste de una fase diaria de isoniazida, rifampicina y pirazinamida por 50 dosis, y una fase bisemanal de isoniazida y rifampicina por 32 dosis. $^{2}$

En este caso, el lactante presentó como complicación una otomastoiditis, por lo que se decidió agregar estreptomicina. La cirugía fue indicada por la necesidad de confirmar el diagnóstico con la macroscopia e histología, para drenar el infiltrado inflamatorio subperióstico, remover el secuestro óseo, descomprimir el nervio facial, y enfrentar en forma adecuada posibles complicaciones $^{9,14}$.

En conclusión, la tuberculosis, es una patología que ha evolucionado junto con los avances de la medicina desde el siglo pasado. De tener incidencias muy elevadas a enfrentarse a las resistencias en su tratamiento, la salud pública mundial ha debido adaptarse a nuevos desafíos para controlarla. La otitis media crónica tuberculosa, es una forma de presentación muy poco habitual, además la similitud de su cuadro clínico con otras etiologías, hace que sea de difícil diagnóstico al no sospechar de ella precozmente. $\mathrm{La}$ realización de un diagnóstico temprano es sumamente importante, ya que mientras antes se trate, menos serán las complicaciones, especialmente la hipoacusia, la cual puede llegar a ser permanente.

Esta revisión tiene como objetivo, despertar en la comunidad médica una inquietud frente a esta condición, ya que en la actualidad, la infección por el bacilo de Köch está aumentando mundialmente, por lo que todo profesional debería estar siempre alerta y preparado para realizar un completo diagnóstico diferencial.

\section{Bibliografía}

1.- NISHIIKE S, IRIFUNE M, OSAKI Y, DOI KATSUMI, KIUCHI N. Tuberculous otitis media: clinical aspect of 12 cases. Ann Otol Rhinol Laryngol 2003; 12: 935-8.

2.- Registro del Programa Nacional de Control de la Tuberculosis 2008. MINSAL. Chile.

3.- AWAN M S, SALAHUDDIN I. Tuberculous otitis media: two case reports and literature review. Ear Nose Throat J 2002; 81: 792-4.

4.- CHIRCH L M, AHMAD K, SPINNER W, JIMÉNEZ V, DONELAN S, SMOUHA E. Tuberculous otitis media: report of 2 cases on Long Island, N.Y., and a review of all cases reported in the United States from 1990 through 2003. Ear Nose Throat J 2005; 84: 488-97.

5.- HERRERA E, CONTRERAS R, VARGAS A. Tuberculosis ótica: comunicación de un caso y revisión bibliográfica. An Orl Mex 2007; 52: 39-43.

6.- VAAMONDE P, CASTRO C, GARCIA N, LABELLA T, LOZANO A. Tuberculosis otitis media: A significant diagnostic challenge. Head Neck Surg 2004; 130: 75966.

7.- SENS P M, ALMEIDA C I, VALLE L O, COSTA L H, ANGELI M L. Tuberculosis of the ear, a professional disease? Braz J Otorhinolaryngol 2008;74: 621-7.

8.- MONGKOLRATTANOTHAI K,ORAM R, REDLEAF M, BOVA J, ENGLUND J. Tuberculous otitis media with mastoiditis and central nervous system involvement. Pediatr Infect Dis J 2003; 22: 453-6.

9.- VITAL V, PRINTZA A, ZARABOUKAS T. Tuberculous otitis media: a difficult diagnosis and report of four cases. Pathol Res Pract 2002; 198: 31-5.

10.- RHO M H, KIM D W, KIM S S, SUNG Y S, KWON J S, LEE S W. Tuberculous Otomastoiditis on High-Resolution Temporal Bone CT: Comparison with Nontuberculous Otomastoiditis with and without Cholesteatoma. Am J Neuroradiol 2007; 28: 493-96.

11.- MUÑOZ A, RUIZ-CONTRERAS J, JIMÉNEZ 
A, MATÉ I, CALVO M, VILLAFRUELA M, et al. Bilateral tuberculous otomastoiditis in an immmunocompetent 5-year-old child: CT and MRI findings. Eur Radiol 2009; 19: 1560-3.

12.- BHALLA R K, JONES T M, ROTHBURN M M, SWIFT A C. Tuberculous otitis media-a diagnostic dilemma. Auris Nasus Larynx 2001; 28: 241-3.
13.- SONMEZ G, TURHAN V, SENOL M G, OZTURK E, SILDIROGLU H O, MUTLU H. Relationship between tuberculous otomastoiditis and tuberculous meningitis. J Laryngol Otol 2008; 122: 893-7.

14.- SAUNDER N C, ALBERT D M. Tuberculous mastoiditis: when is surgery indicated? Int J Pediatr Otorhinolaryngol 2002; 65: 59-63.

Correspondencia a:

Juan I. Juanet G.

El Director 5813 Dpto. 43 Las Condes, Santiago. Chile.

Teléfono fijo: 453-7416 Teléfono Cel.: 98835363

E-mail: jijuanet@gmail.com 